\title{
SEVERE GRANULAR DYSTROPHY: A PEDIGREE WITH PRESUMED HOMOZYGOTES
}

\author{
CHARLES J. M. DIAPER \\ Bradford
}

\begin{abstract}
SUMMARY
Three siblings with severe granular corneal dystrophy are described. They are most likely to be homozygous for the dominantly inherited gene. They are offspring of a consanguineous marriage, with both parents affected with corneal dystrophies. The case reports describe the severe course of this condition, requiring multiple grafting procedures. Clinical pictures of the family are shown.

Granular dystrophy is a corneal condition of uncertain pathogenesis with no extraocular features. It is an autosomal dominant corneal dystrophy with high penetrance. ${ }^{1}$

Clinically the condition presents usually in the thirties or forties with sugar-granule-like opacities in the anterior or middle corneal stroma. Symptoms, which are usually a slight decrease in vision and a foreign body sensation, may be variable but tend to be consistent within a family. ${ }^{2}$ Visual function is maintained until later in life when a corneal graft may be required.

There is, however, a more severe variant which presents earlier and has a greater effect on visual acuity. The case histories of a pedigree with both variants is reported.
\end{abstract}

\section{CASE REPORTS}

\section{Case 1}

This 14-year-old Asian boy presented in 1970. He had had episodes of sore watery eyes. On examination he had a granular corneal dystrophy and nystagmus. The fundus was normal and there was no afferent pupillary defect. Visual acuity was $6 / 18$ in the right eye and 6/36 in the left with contact lenses.

Five years later, when his best corrected visual acuity had decreased to $6 / 60$ in both eyes, he underwent bilateral lamellar corneal grafts. Best visual acuity after surgery was $6 / 18$ right and 6/24 left. There is no record of the histopathological findings at that time.

By 1983, however, the visual acuity had again dropped

Correspondence to: Mr Charles J. M. Diaper, FRCS(Ed), FRCOphth, 4/2, 15 Clarendon Street, Glasgow G20 7QP. to 6/60 right and counting fingers (CF) left, and a left penetrating graft was performed. Histological examination confirmed granular dystrophy mainly in the superficial stroma. Best recorded visual acuity in the left eye after grafting was 6/24. In 1987 the right eye, with a visual acuity of $\mathrm{CF}$, was grafted. Histopathological examination confirmed the earlier report. Best visual acuity was $6 / 12$ following surgery.

In 1988, an early recurrence was noted in the right grafted tissue and vision on the left had been reduced to CF by recurrence. The left eye was regrafted that year and the right in 1990, when vision again had been reduced to CF. Best visual acuity in the right eye was $6 / 18$.

However, in 1993 his visual acuity has fallen due to recurrence (Fig. 1). He has had left photokeratectomy to avoid further grafting procedures. Early corrected visual acuity is $6 / 36$.

\section{Case 2}

This 8-year-old boy was seen with his brother in 1970 . Clinically he had granular dystrophy and his vision was $6 / 6$ in the right eye and $6 / 18$ in the left. At the age of 18 years, with vision reduced to $6 / 60$ in each eye, he had his right eye grafted. The best acuity after surgery was $6 / 6$. The left eye was grafted in 1981 and histology confirmed a superficial granular dystrophy. At this time recurrence was noted in the right graft, which was regrafted in 1986 when vision was reduced to $\mathrm{CF}$.

By 1988, the dystrophy had recurred in the right eye and visual acuities were $6 / 12$ right and $6 / 9$ left. There has been further visual deterioration (Fig. 2) and in 1993 left photokeratectomy was performed. Early corrected visual acuity was $6 / 12$.

\section{Case 3}

This girl presented in 1973 , at the age of 10 years, with a superficial central dystrophy. Her general practitioner's referral letter contained a family tree indicating that her father was also affected. Visual acuities at the time were $6 / 18$ in the right eye and $6 / 5$ in the left. 


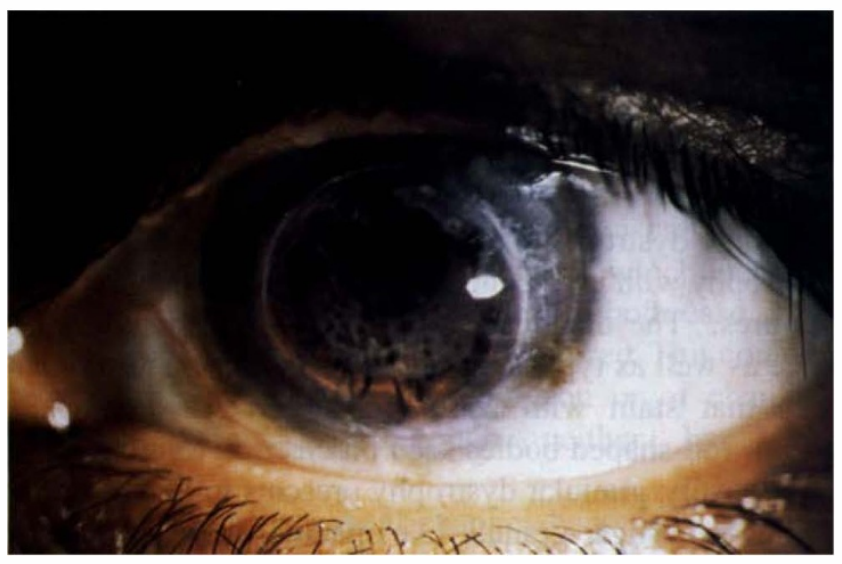

Fig. 1. Left eye of case 1 .

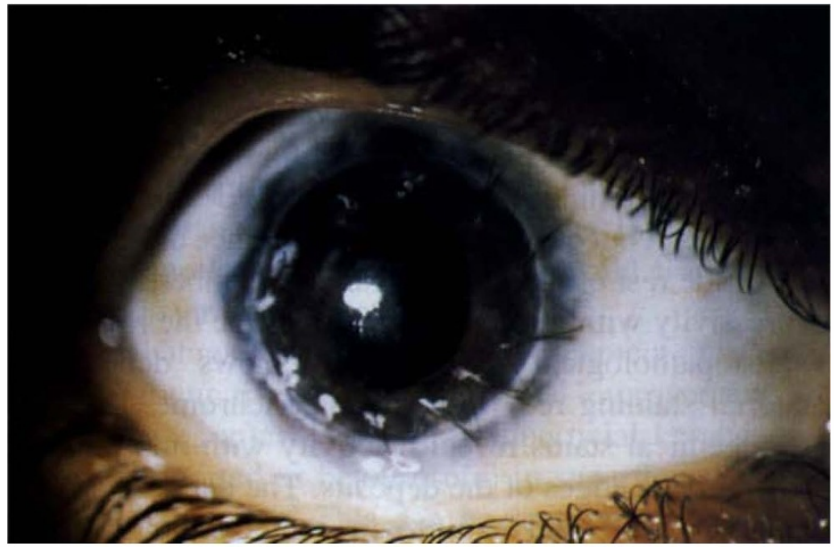

Fig. 3. Right eye of case 3. Note the recurrence at suture sites.

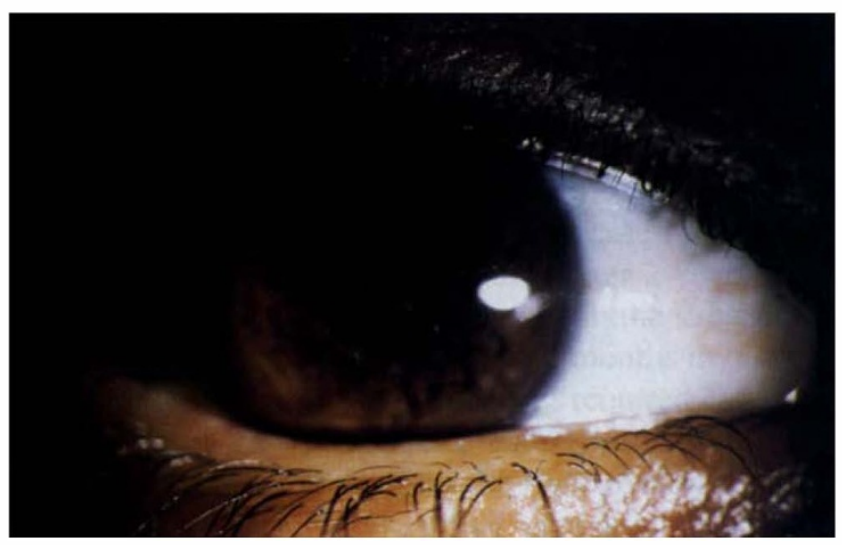

Fig. 5. Left eye of eldest asymptomatic sibling.

By 1978 her visual acuities had dropped to 6/36 in each eye and she underwent a left penetrating graft. Histological examination confirmed granular dystrophy. In 1979 early recurrence was noted and in 1980 a right penetrating graft was performed. Vision after surgery was $6 / 24$ in the right eye and $6 / 36$ in the left.

In 1983 a left lamellar graft was performed. Histological examination confirmed superficial granular dystrophy. By the end of the year the dystrophy had recurred. With vision now reduced to $\mathrm{CF}$ she underwent a left penetrating graft in 1984. Recurrence was noted in the right graft at the

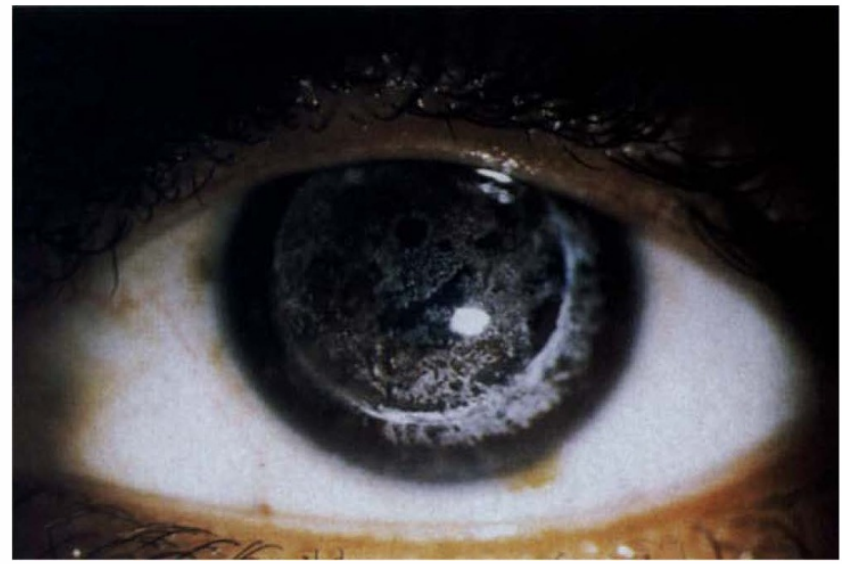

Fig. 2. Left eye of case 2 .

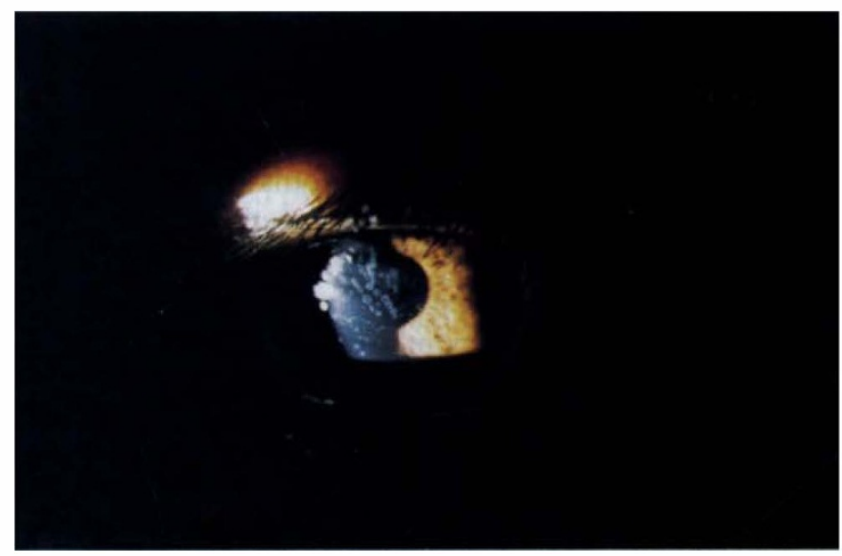

Fig. 4. Right eye of case 4.

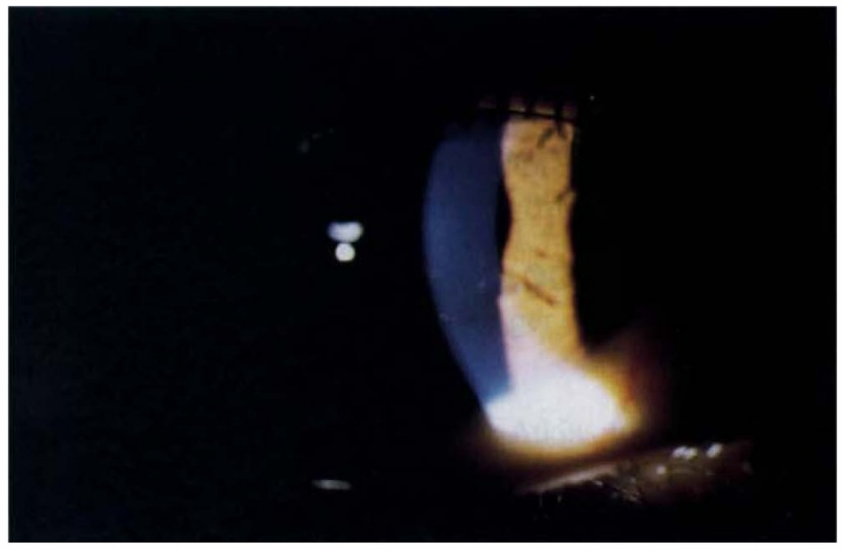

Fig. 6. Right eye of second youngest sibling, with early granules. end of 1984 and in the left at the end of 1985 . Best acuity of $6 / 18$ was achieved in the left.

However, in 1990 with vision reduced to $\mathrm{CF}$ in the right eye, she underwent a right penetrating graft. In 1991 a recurrence was noted in the right eye (Fig. 3).

\section{Case 4}

The mother of the above patients was referred by her optician, in 1982, at the age of 49 years. Her visual acuities were $6 / 6$ in both eyes and clinically she had typical granular dystrophy. In 1986 her vision had reduced in 6/60 in 


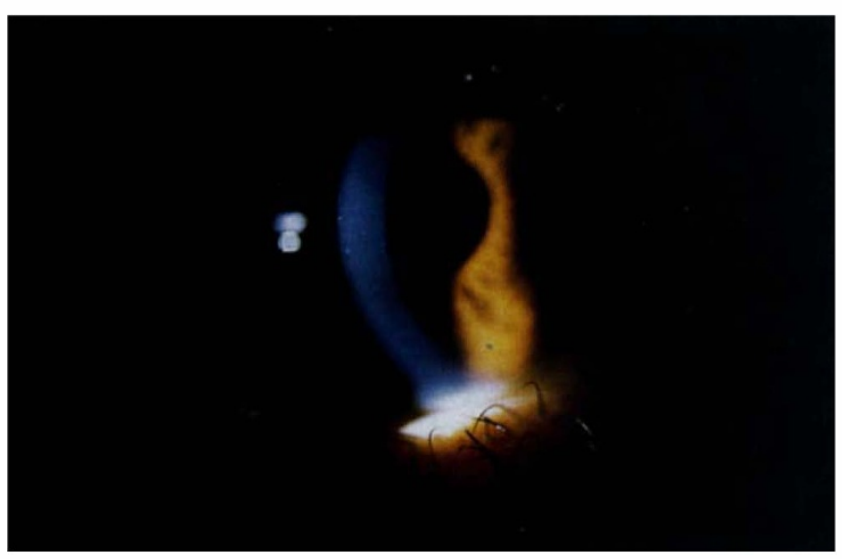

Fig. 7. Right eye of youngest sibling, with early granules.

each eye and she was listed for grafting; she later removed herself from the list.

In 1992 she was again referred by an optician for assessment of her corneal dystrophy. Her vision at this time was $6 / 18$ in each eye and her chief symptoms were of recurrent erosion. She is being treated symptomatically (Fig. 4).

Both case 1 and case 3 have been put on the partially sighted register. Their poor vision necessitated their attendance at a school for the visually impaired. The rereferral of case 4 prompted a review of all family members.

The referral letter of case 3 indicates that the father, who died in India in 1975, also had a corneal dystrophy. The records suggest that this was a lattice dystrophy. There is no clear ophthalmic record of the father.

The family review revealed that the second eldest son (34 years old) had an asymptomatic granular dystrophy (Fig. 5) similar to his mother's. His visual acuities were $6 / 4$ in both eyes. The two youngest of this generation had early superficial lesions suggestive of granular dystrophy (Figs. 6,7). The third generation were all between 1 and 7

\section{Phenotype

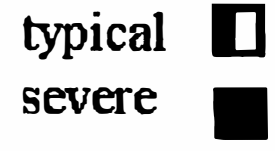

years old, and had no evidence of corneal disease. The pedigree is outlined in Fig. 8. There are no other members of this family in the country.

\section{DISCUSSION}

Granular dystrophy is an autosomal dominant corneal dystrophy with high penetrance. There are no extraocular features. ${ }^{1}$ The diagnostic criteria are: dominant inheritance as well as typical slit lamp appearance and/or granules that stain with Masson trichrome histologically and/or rod-shaped bodies seen on electron microscopy. ${ }^{2}$

Typically, granular dystrophy presents in the thirties or forties with sugar-granule-like opacities in the anterior or mid stromal cornea. The intervening spaces are clear. In Moller's review of Danish pedigrees, ' a third were aware of their condition before the age of 15 years, the rest before 45 years. Diagnostic corneal changes were visible by 5 years old. Symptoms are a gradual decrease in vision and recurrent erosions. These can be variable but tend to be consistent within a pedigree. In Moller's review of 140 patients, the clinical appearance ranged from mild to 'monstrous', but only 1 patient's appearance differed from that of the rest of the affected family members. Constant expressivity within a family appears to be the rule. ${ }^{2}$

Histopathological examination ${ }^{3}$ shows deposits of material staining red with Masson trichrome. Immunohistochemical stains reveal reactivity with microfibrillar protein at the edges of the deposits. The deposits are rich in phospholipids and their protein composition is not suggestive of a collagenous origin. Electron microscopy shows electron-dense rods in an amorphouse matrix.

Unusually severe cases have been reported..$^{4-6}$ These have presented much earlier and have had a more severe course necessitating multiple grafting procedures by early adulthood. These patients have been, in as far as it has been possible to ascertain, the offspring of consanguineous marriages. Clinically the granules have been more

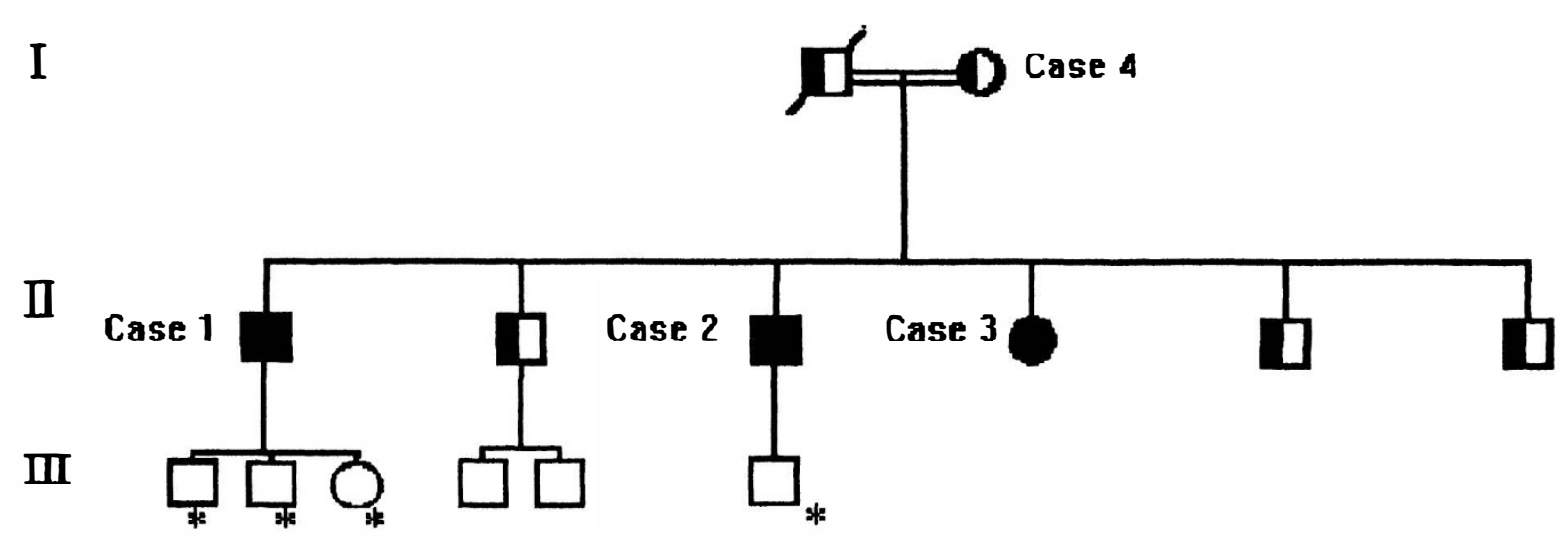

Fig. 8. Family pedigree. Asterisks indicate obligate heterozygotes. 
superficial and combined with the earlier age of onset; the term 'superficial juvenile granular dystrophy' has been proposed. ${ }^{6}$

In typical granular dystrophy vision is not affected until the thirties or forties, though severe handicap is rare until after working age.' The reduction in visual acuity is mainly the result of corneal irregularity, which increases with the amount of corneal change.' In both case 2 and case 4 a marked variation of vision occurred. This could be related to episodes of corneal erosion or of changing irregular astigmatism, though neither has been documented.

Early diagnostic corneal changes have been noted by the age of 5 years in affected individuals.' though some patients only have minimal signs at 16 years old. In this family there are four members of the third generation that are obligate heterozygotes. Their ages range from 1 to 7 years and at present they show no clinical evidence of granular dystrophy. It can be expected that the clinical course of all the heterozygotes in this family will be similar and that these four will not develop signs until their late teens. It is interesting to note that Fig. 5 and the figures in Moller's paper ${ }^{2}$ described as mild cases, which developed symptoms later, look alike.

Corneal abrasion may be a useful treatment in selected patients, giving rise to subjective visual improvement, but this decreases after 2-3 years.' Superficial keratectomy has been reported as being useful in superficial juvenile granular dystrophy. ${ }^{6}$ This can be repeated as the endothelium is undisturbed and does not require donor material. Penetrating grafts have been the preferred option.

As in other dystrophies there is a high incidence of graft recurrence. In Moller's review, all of 14 grafts had recurred by 7 years.' The time to regrafting had not been evaluated, but was thought to be in the region of 10 years.? In previously reported severe cases, graft recurrence has been rapid, leading to multiple grafts. ${ }^{4}$ In case 1 recurrence after initial lamellar grafts was noted after 2 years, with visual failure after 5 years. Recurrence in the subsequent penetrating grafts was apparent after 6 months in one eye and 2 years in the other. Regrafting was required $3-5$ years after the initial grafts. In case 2 recurrence was noted 18 months after initial grafting in the right eye, which was regrafted 6 years after the initial operation. In case 3, initial grafting was performed with recurrence after 2 years. A subsequent lamellar graft showed recurrence after 6 months with regrafting a year later.

In the notes of case 3, the GP's referral included a family tree indicating that the father was affected by a corneal dystrophy. This was noted as being lattice dystrophy at the initial consultation while the daughter was diagnosed as having granular dystrophy. However, lattice and granular dystropies may have very similar clinical appearances and the true diagnosis is not made until a histological examination is performed. ${ }^{8}$ The histological difference is that in lattice dystrophy there are characteristic deposits of amyloid not found in granular dystrophy. In none of the offspring has amyloid been demonstrated in host corneal buttons. As the marriage was consanguineous, it is most likely that the partners shared the same dystrophy.

The severely affected patients reported in the literature ${ }^{4}$ and the cases described here probably represent homozygotes for granular dystrophy. Some of the pedigrees reported by Sajjadi and Javadi ${ }^{6}$ also indicate homozygotes; however, the authors point out the unreliability of these data. No increased abortion rates have been noted. This mitigates against a gross chromosomal abnormality or translocation. A linkage study ${ }^{10}$ found a lodscore of 1.04 in females to the CIR system. This is localised to chromosome 12p13. This lodscore is below the commonly accepted value of 3 but does give an indication for further study. The cases reported here were monosymptomatic with no other features detected. There have been no cases of stillbirth or miscarriage in this family, which suggests that there is no associated chromosomal translocation in this pedigree. This pedigree is too small for practical crosslinkage studies; however, if taken with other documented pedigrees, such studies would open the door for the gene location of granular dystrophy.

In other dominant conditions, surviving homozygotes show not only more severe signs but also additional signs in tissues not normally involved.? In this pedigree, and other reports, the cornea is the only site of disease. This suggests that the gene responsible is controlling a process peculiar to the cornea, possibly in the processing of epithelial proteins or phospholipids. ${ }^{3}$

I believe this family represents a three-generation pedigree with seven clinically affected members, three of whom are homozygotes. This is rare both in practice and in the literature. ${ }^{4}$ It supports previous case studies indicating that homozygous patients present earlier, in a more severe form and have earlier recurrences following surgical intervention. However, because of the lack of documentation on the father, this conclusion is presumptive. If this is the case, then in generation III, four are obligate heterozygotes, who will manifest with slit lamp evidence of granular dystrophy, like their younger uncles, in their late teens.

I wish to thank $\mathrm{Mr}$ Hopkins and $\mathrm{Mr}$ Atkins for permission to report their patients and for their support. My thanks to Sandra Smallwood and the Departments of Medical Illustration at Bradford Royal Infirmary and Saint James' University Hospital for the photography, and illustration.

Key words: Genetics, Granular corneal dystrophy, Homozygotes.

\section{REFERENCES}

1. Moller HU. Granular corneal dystrophy Groenouw type 1. Clinical aspects and treatment. Acta Ophthalmol (Copenh) 1990;68:384-9.

2. Moller HU. Inter-familial variability and intra-familial similarities of granular corneal dystrophy Groenouw type 1 with respect to biomicroscopical appearance and symptomatology. Acta Ophthalmol (Copenh) 1989;67:669-77.

3. Rodriques MM, Krachmer JH. Recent advances in corneal stromal dystrophies. Cornea 1988;7:19-29.

4. Moller HU, Ridgway AE. Granular corneal dystrophy Groe- 
nouw type 1: a report of a probable homozygous patient. Acta Ophthalmol (Copenh) 1990;69:97-101.

5. Roderigues MM, Gaster RM, Pratt MV. Unusual superficial confluent form of granular corneal dystrophy. Ophthalmology 1983;90:1507-11.

6. Sajjadi SH, Javadi MA. Superficial juvenile granular dystrophy. Ophthalmology 1992;99:95-102.

7. Moller HU. Granular dystrophy Groenouw type 1: clinical and genetic aspects. Acta Ophthalmol (Suppl) 1991; 198:1-40.

8. Folberg AA, Croxatto JO, Driezen NG, Panjwani N, Laib- son PR, Boruchoff SA, et al. Clinically atypical granular corneal dystrophy with pathologic features of lattice-like amyloid deposits: a study of these families. Ophthalmology 1988;95:46-51.

9. Moller HU. Granular corneal dystrophy Groenouw type 1: 115 Danish patients. An epidemiological and genetic population study. Acta Ophthalmol (Copenh) 1990;68:297-303.

10. Moller HU, Eiberg H, Kruse TA. Linkage relations of the locus for granular corneal dystrophy Groenouw type 1 with 35 polymorphic systems. Acta Ophthalmol (Copenh) 1989;67:721-3. 